\title{
Non-Proteinuric Preeclampsia: Prevalence, Factors Associated and End-Organ Dysfunction at Mbarara Regional Referral Hospital in Southwestern Uganda
}

Asiphas Owaraganise ( $\nabla$ asiphas@gmail.com )

Mbarara University of Science and Technology

Wasswa GM Ssalongo

Mbarara National Referral Hospital

Leevan Tibaijuka

Mbarara National Referral Hospital

Musa Kayondo

Mbarara University of Science and Technology

Godfrey Twesigomwe

Mbarara University of Science and Technology

Joseph Ngonzi

Mbarara University of Science and Technology

Henry M Lugobe

Mbarara University of Science and Technology

\section{Research Article}

Keywords: Pregnancy, Non-proteinuric, Proteinuric, Preeclampsia, End-organ, Dysfunction, Mbarara.

Posted Date: March 1st, 2021

DOl: https://doi.org/10.21203/rs.3.rs-242190/v1

License: (9) (i) This work is licensed under a Creative Commons Attribution 4.0 International License. Read Full License 


\section{Abstract}

Background: Formerly, preeclampsia was only diagnosed if high blood pressure and proteinuria were present in a gravid woman at or past 20 gestation weeks. Although it is possible to have preeclampsia yet never have proteinuria, the literature on how common and factors associated with non-proteinuric preeclampsia as well as whether the frequency of end-organ dysfunction among women with nonproteinuric differs from those with proteinuric pre-eclampsia is scant.

Objectives: To determine the prevalence of, factors associated with non-proteinuric preeclampsia, and compare the frequency of end-organ dysfunction between women with non-proteinuric and proteinuric preeclampsia.

Methods: Between November 2019 and May 2020, women with pre-eclampsia were consecutively enrolled in a cross-sectional study at Mbarara Regional Referral Hospital. We interviewed all pregnant women $\geq 20$ gestation weeks presenting with hypertension $\geq 140 / 90 \mathrm{mmHg}$ and recorded their sociodemographic, medical, and obstetric characteristics. We excluded women with chronic hypertension and pregnancy hypertension. We measured bedside proteinuria on clean-catch urine, complete blood count, and renal function and liver enzyme tests. Pre-eclampsia was defined as hypertension plus any feature of severity including $<100,000$ platelets $/ \mathrm{ul}$, creatinine $>1.1 \mathrm{~g} / \mathrm{dl}$, and liver transaminases $\geq$ twice upper normal limit with or without proteinuria. We computed the proportion of total participants with nonproteinuric pre-eclampsia at $\leq+2$ urine dipstick cut-off, determined the factors associated with nonproteinuric preeclampsia using logistic regression; and used Chi-square test to compare the frequency of end-organ dysfunction between non-proteinuric preeclampsia and proteinuric groups.

Results: We enrolled 134 of the screened 136 participants. Their mean age was 26.9 (SD \pm 7.1 ) years and $51.5 \%$ were primigravid. The prevalence of non-proteinuric preeclampsia was $24.6 \%$ (95\% Cl: 17.9-32.7). Primigravidity (aOR 2.70 95\% Cl:1.09-6.72, p=0.032) was the factor independently associated with nonproteinuric pre-eclampsia. There was a non-significant difference in the frequency of end-organ dysfunction in women with non-proteinuric and proteinuric pre-eclampsia, $\mathrm{p}=0.309$.

Conclusion: Non-proteinuric preeclampsia is common, especially among primigravidae. End-organ dysfunction occurs similarly in women with non-proteinuric and proteinuric preeclampsia. Obstetrics care providers should emphasize laboratory testing, beyond proteinuria, among all women with hypertensive disorders of pregnancy to optimally diagnose and manage non-proteinuric preeclampsia.

\section{Synopsis}

Non-proteinuric preeclampsia is common and associated with primigravidity. Since women suffer similar end-organ dysfunction, proteinuria testing alone is insufficient to diagnose their pre-eclampsia syndrome.

\section{Background}


The global prevalence of pre-eclampsia ranges from eight to ten per cent [1], and it is the third leading cause of maternal mortality in southwestern Uganda [2]. Formerly, pre-eclampsia diagnosis was based on pregnancy hypertension of at least $140 / 90 \mathrm{mmHg}$ at or past 20 weeks of gestation and mandatory proteinuria $[3,4]$ measured commonly as a random urine protein to creatinine ratio $\geq 0.3$ or persistent $\geq$ $30 \mathrm{mg} / \mathrm{dL}$ or $\geq 2+$ on the dipstick, and rarely as a 24 -hour urinary excretion $>300 \mathrm{mg}[5]$. However, preeclampsia with features of severity can indeed occur in asymptomatic women and severe maternofoetal adverse events may happen before the traditional proteinuria-based criteria are met [6-8]. While optimally identifying and treating women with pre-eclampsia reduces and averts morbidity and mortality from hypertensive disorders in pregnancy (HDP) $[9,10]$, the heterogeneity in clinical presentation [11] and clinical practice guidelines [12] delays diagnosis in settings without readily available hematologic testing -especially when overt proteinuria is absent [13-15].

To optimize surveillance, diagnosis and delivery for improved outcomes of women with pre-eclampsia, their risk profile like sociodemographic, medical, and obstetric factors must be known $[16,17]$. Since such factors, as well as management protocols that influence outcomes of the entire pre-eclampsia care cascade [18-21], are setting-specific, low-income contextual data are needed to guide comprehensive screening of all pregnant women to timely diagnose preeclampsia. Moreover, most studies report nonproteinuric pre-eclampsia in developed countries[22, 23] yet the developing counterparts suffer seven times HDP incidence and over $99 \%$ of associated fetal-maternal deaths [23-25]. We enrolled pregnant women diagnosed with pre-eclampsia at a tertiary hospital in southwestern Uganda to determine the prevalence and factors associated with non-proteinuric preeclampsia and compare the frequency of endorgan dysfunction between women with non-proteinuric and proteinuric preeclampsia.

\section{Materials And Methods}

\section{Study design and setting}

We conducted a cross-sectional study of women with pre-eclampsia admitted to the antenatal ward of Mbarara Regional Referral Hospital (MRRH) in southwestern Uganda from November 2019 to May 2020. MRRH is a government-funded public tertiary hospital. The records show that the hospital conducted approximately 9,000 deliveries for the calendar year 2019 . The hospital's maternal mortality ratio stands high at 261 per 100,000 live births [25] with pre-eclampsia among top three causes[2].

\section{Study participants}

We studied all pregnant women, including emancipated minors under the age of 18 years diagnosed with pre-eclampsia. We defined preeclampsia as de novo and sustained hypertension ( $\geq 140 / 90 \mathrm{mmHg})$ at $\geq$ 20 weeks of gestation with proteinuria or at least one feature of end-organ dysfunction for those without proteinuria. 


\section{Eligibility criteria}

We included gravid women at or past 20 weeks of gestation with hypertension and proteinuria or at least one severity feature of pre-eclampsia for those without proteinuria. We excluded women with chronic hypertension and gestational hypertension.

\section{Sample size estimation and sampling procedure}

All pregnant women diagnosed with preeclampsia between November 2019 and May 2020 at MRRH were consecutively screened and enrolled in the survey without a priori-determined sample size.

\section{Study Procedures}

Research staff consecutively approached all pregnant women who presented to the antenatal unit of the maternity ward of MRRH, explained the study approach and invited them to participate. Of women who accepted to be screened, those that passed the study eligibility criteria were enrolled in the study. To identify those with hypertension, all pregnant women presenting at or above 20 weeks of gestation, had a screening blood pressure measured by research staff on admission. Our blood pressure measurement standard operating procedure was as follows: We used a Visomat@ Electronic digital dial blood pressure machine (manufactured byOBL: Visomat, Zum Ottersberg Wertheim am Main, GERMANY) to measure the blood pressure. A woman was allowed time to rest seated without talking for at least 5 minutes. Blood pressure cuff was placed $1-2 \mathrm{~cm}$ above the elbow on either arm supported at heart level-on a table or a chair armrest while she was seated leaning back against a chair, without tight clothing around the upper arm and both feet on the floor. Women were instructed not to move, strain or talk while the measurement was being taken. The machine was turned on and the cuff allowed to automatically inflate, deflate and the display result was taken as the correct woman's blood pressure-the process was repeated upon displaying an 'error' reading. We defined hypertension as sustained elevated blood pressure that is, systolic blood pressure $\geq 140 \mathrm{mmHg}$ and/or diastolic blood pressure $\geq 90 \mathrm{mmHg}$ four-hours apart or single reading of severe hypertension is defined as systolic blood pressure $\geq 160 \mathrm{mmHg}$ and/or diastolic blood pressure $\geq 110 \mathrm{mmHg}$ or documented antihypertensive medication use to control the blood pressure via woman's medical records review.

Each woman who screened positive for hypertension parallelly underwent further clinical evaluation and laboratory screening-full hemogram, renal function test and liver enzymes test and bedside proteinuria measurement to diagnose pre-eclampsia. The clinical evaluation included history taking, physical examination and medical records (antenatal card) review.

To measure spot proteinuria, the research assistant gave each woman a uniquely labelled sterile urine container and instructed her to collect about $10 \mathrm{mls}$ of clean-catch urine or collected it at urine drainage port after removing urine bag for those who had urethral catheter in-situ. Research assistant immersed a 
Cypress Diagnostics ${ }^{\circledR}$ colour-coded 10 parameter urine dipsticks (manufactured by Cypress Diagnostics, Hulshout, Belgium) in the sample for 60 seconds and then compared to container visual colour codes following the manufactures instructions on the package insert. The read off result was further classified as non-proteinuric if $\leq+1$ and proteinuria if $\geq+2$.

For hematologic tests, ten millilitres of blood sample were drawn from an easily accessible vein on the arm and halved into EDTA and plain vacutainers. These samples with complete laboratory request forms were taken to MRRH laboratory for full hemogram, liver enzymes and renal function testing. The Humastar-200 ® clinical chemistry analyser (manufactured by HUMAN Biochemica und Diagnostica $\mathrm{GmbH}$, Wiesbaden, Germany) was used to measure the serum creatinine in $\mathrm{mg} / \mathrm{dL}$, liver transaminases in $\mathrm{IU} / \mathrm{L}$ on the $5 \mathrm{mls}$ blood in plain vacutainer. The other $5 \mathrm{mls}$ in EDTA vacutainer bottle was run in the Sysmex XN-1000i ${ }^{\circledR}$ 5-part haematology analyser (manufactured by Sysmex America, Inc. Lincolnshire, Illinois, USA) to measure haemoglobin concentration in $\mathrm{g} / \mathrm{dL}$ and platelet count per microliter.

We diagnosed pre-eclampsia if a gravid woman at or past 20 weeks of gestation had hypertension and proteinuria-proteinuric pre-eclampsia or hypertension and at least one severity feature of pre-eclampsia if without proteinuria-non-proteinuric pre-eclampsia. We defined end-organ dysfunction as presence of at least any one of the following in a woman who screened positive for pre-eclampsia: 1) unexplained severe headache or blurred vision or coma or convulsion-cerebral dysfunction; liver transaminases level at least twice of the normal upper limit-hepatic dysfunction; 3) thrombocytopenia $<100,000 / \mu \mathrm{L}-$ hematologic dysfunction; 4) serum creatinine > $1.1 \mathrm{mg} / \mathrm{dL}$-renal dysfunction; and, 5) multi-organ dysfunction if $\geq 1$ organ dysfunction was diagnosed concomitantly in one woman.

\section{Data collection}

We interviewed women with pre-eclampsia (abstracted some of the data from the woman's clinical record) and captured the data using a pretested-structured questionnaire. The variables recorded included: 1) socio-demographic data -age, level of education, marital status, employment status, age, occupation, alcohol use or smoking; 2) clinical data- chronic hypertension, renal disease, liver disease; unexplained severe headache, visual disturbances, convulsions, epigastric pain, dyspnoea, plus weight and height measurements; 3) obstetric data-parity, gestational age, multifetal gestation, history of preeclampsia during previous pregnancies, antenatal attendance and booking blood pressure; and 4) blood test results data-full hemogram, renal function and liver transaminases.

\section{Data analysis}

Data were then entered into a secure online backed up $\mathrm{RECaP} B[26]$ database version 8.2 hosted at Department of Obstetrics and Gynecology at Mbarara University of science and Technology (MUST). Statistical analyses were performed using Stata Statistical Software: Release 14 (StataCorp LP, College Station, Texas USA). 
We computed descriptive statistics and tabulated the baseline participant characteristics as frequency proportions and percentages for categorical variables, or mean with standard deviation for continuous variables-compared across the non-proteinuric and proteinuric groups. The prevalence of non-proteinuric pre-eclampsia was determined as a proportion of participants with non-proteinuria and expressed as a percentage. We used binary logistic regression to determine sociodemographic, obstetric, clinical and laboratory variables associated with dependent variables. Independent variables with at $p<0.2$ were entered into a multivariable logistic-regression model to analyse factors independently associated with non-proteinuric preeclampsia using adjusted OR at $95 \%$ confidence interval, $p<0.05$. To compare the frequency of end-organ dysfunction, Pearson's chi-squared test statistic $(\chi 2)$ was calculated for women with non-proteinuric and proteinuric pre-eclampsia, at one degree of freedom and $p<0.05$.

\section{Ethical consideration}

Ethical approval to conduct this study was obtained from the Mbarara University Research Ethics Committee (Protocol reference number 14/01-19). Written informed consent was obtained from all study participants using prior-approved consent forms in English and local language translated versions. Informed consent was obtained from all adults aged 18 years and over independently while parent or legal guardian gave informed consent for study participants below 18 years of age.

\section{Results}

A total of 136 pregnant women with pre-eclampsia were admitted to the antenatal ward at Mbarara Regional Referral Hospital. Two declined to participate. One hundred thirty-four (134) were enrolled in the study and their data analysed. Participants' baseline characteristics are shown in Table 1. The mean age of the enrolled participants was 26.9 (SD \pm 7.1 ) years. Women with non-proteinuric pre-eclampsia were different from their counterparts with proteinuric preeclampsia in terms of age and parity. They tended to be younger, the mean age of $23.7 \pm 6.8$ years versus $27.1 \pm 7.1, p=0.019$, and primigravidae, $72.7 \%(n=24)$ versus $44.55 \%(n=45)$ respectively, $p=0.005$.

Table 1. Baseline characteristics of women with preeclampsia at Mbarara Regional Referral Hospital 


\begin{tabular}{|c|c|c|c|c|}
\hline Variable $N=134$ & Total n (\%) & $\begin{array}{l}\text { Non-proteinuric } \\
\text { pre-eclampsia } \\
(\mathrm{n}=33)\end{array}$ & $\begin{array}{l}\text { Proteinuric } \\
\text { pre-eclampsia } \\
(n=101)\end{array}$ & $\mathrm{p}$-value \\
\hline Age, years (mean $\pm S D$ ) & $26.9 \pm 7.1$ & $23.7 \pm 6.8$ & $27.1 \pm 7.1$ & $0.019 *$ \\
\hline Married & & & & 0.153 \\
\hline No & $09(6.7)$ & $29(87.88)$ & $96(95.05)$ & \\
\hline Yes & 125(93.3) & $04(12.12)$ & $05(4.95)$ & \\
\hline Occupation & & & & 0.476 \\
\hline Housewife & $76(56.7)$ & $17(51.52)$ & $59(58.42)$ & \\
\hline Employed & $48(35.8)$ & $4(12.12)$ & $06(5.94)$ & \\
\hline Business & $10(7.5)$ & $12(36.36)$ & $36(35.64)$ & \\
\hline Education & & & & 0.080 \\
\hline$\leq$ Primary & $85(63.4)$ & $15(45.45)$ & $28(27.72)$ & \\
\hline Secondary & $43(32.1)$ & $15(45.45)$ & $70(69.31)$ & \\
\hline$\geq$ Tertiary & $06(4.5)$ & 03(9.09) & $03(2.97)$ & \\
\hline Referred in & & & & 0.102 \\
\hline Yes & $85(63.4)$ & $17(51.52)$ & $68(67.33)$ & \\
\hline No & $49(36.57)$ & $16(48.48)$ & $33(32.67)$ & \\
\hline Parity & & & & $0.005^{\star}$ \\
\hline Primigravidity & $69(51.5)$ & $24(72.73)$ & $45(44.55)$ & \\
\hline Multiparous & $65(48.5)$ & $9(27.27)$ & $56(55.45)$ & \\
\hline Weeks of gestation & & & & 0.274 \\
\hline$<37$ & $70(52.2)$ & $14(42.42)$ & $56(55.45)$ & \\
\hline$\geq 37$ & $64(47.8)$ & 19(57.58) & $45(44.55)$ & \\
\hline Presenting symptom & & & & 0.319 \\
\hline Headache & 102(76.1) & $23(69.70)$ & $79(78.22)$ & 0.319 \\
\hline Epigastric pain & $87(64.9)$ & $18(54.55)$ & $69(68.32)$ & 0.150 \\
\hline Blurred vision & $57(42.5)$ & $14(42.42)$ & $43(42.57)$ & 0.988 \\
\hline Fit/Eclampsia & $15(11.2)$ & $04(12.12)$ & 11(10.89) & 0.846 \\
\hline
\end{tabular}




$\begin{array}{llll}<4 \text { times } & 72(53.7) & 5(27.78) & 74(27.82) \\ \geq 4 \text { times } & 62(46.3) & 13(72.22) & 192(72.18)\end{array}$

Pre-eclampsia history

$\begin{array}{lll}\text { Yes } & 09(6.8) & 04(12.12)\end{array}$

Booking Blood Pressure $(\mathrm{mmHg})$

$\begin{array}{llll}<130 / 80 & 58(43.28) & 12(36.36) & 46(45.54) \\ \geq 130 / 80 & 76(56.72) & 21(63.64) & 55(54.46)\end{array}$

Admission Blood Pressure(mmHg)

0.109

$\begin{array}{llll}<160 / 110 & 65(48.51) & 13(39.39) & 52(51.49) \\ \geq 160 / 110 & 69(51.49) & 20(60.61) & 49(48.51)\end{array}$

Serum Creatinine $\mathrm{mg} / \mathrm{dL}$

$\begin{array}{llll}\leq 1.09 & 100(74.63) & 25(75.76) & 75(74.26) \\ \geq 1.10 & 34(25.37) & 8(24.24) & 26(25.74)\end{array}$

Transaminases IU/L

$\begin{array}{llll}<80 & 96(71.64) & 24(72.73) & 72(71.29) \\ \geq 80 & 38(28.36) & 9(27.27) & 29(28.71)\end{array}$

Platelet count per uL

0.664

$\begin{array}{llll}\geq 100000 & 109(81.34) & 26(78.79) & 83(82.18) \\ <100000 & 25(18.66) & 07(21.21) & 18(17.82)\end{array}$

The prevalence of non-proteinuric preeclampsia was 24.6 per cent $(95 \% \mathrm{Cl}: 17.9-32.7)$, as shown in figure 1.

On multivariable logistic regression, only primigravidity (aOR $2.7095 \% \mathrm{Cl}: 1.09-6.72, \mathrm{p}=0.032$ ) remained independently associated with non-proteinuric pre-eclampsia (Table 2).

Table 2 Crude and adjusted odds ratios of factors associated with non-proteinuric preeclampsia at Mbarara Regional Referral Hospital 


\begin{tabular}{|c|c|c|c|c|c|c|}
\hline Factors $\mathrm{N}=134$ & Category & $\begin{array}{l}\text { Non-PPE } \\
\text { n (\%) }\end{array}$ & cOR $(95 \% \mathrm{Cl})$ & $\begin{array}{l}\mathrm{p}- \\
\text { value }\end{array}$ & aOR $(95 \% \mathrm{Cl})$ & $\begin{array}{l}\mathrm{p}- \\
\text { value }\end{array}$ \\
\hline \multirow[t]{3}{*}{ Age (Years) } & $20-30$ & $12(36.36)$ & 1 & & Referent & \\
\hline & $15-19$ & $14(42.42)$ & $\begin{array}{l}2.9(1.11- \\
8.37)\end{array}$ & 0.040 & $\begin{array}{l}2.19(0.81- \\
5.02)\end{array}$ & 0.059 \\
\hline & $>30$ & $7(21.22)$ & $1.2(0.41-3.28)$ & 0.770 & $\begin{array}{l}0.67(0.22- \\
2.05)\end{array}$ & 0.479 \\
\hline \multirow[t]{2}{*}{ Gravidity } & Multigravida & $9(27.27)$ & 1 & & Referent & \\
\hline & Primigravida & $24(44.55)$ & $\begin{array}{l}3.31(1.40- \\
7.84)\end{array}$ & 0.020 & $\begin{array}{l}2.70(1.09- \\
6.72)\end{array}$ & $0.032^{\star}$ \\
\hline \multirow[t]{2}{*}{ Referred in } & No & 16(18.18) & 1 & & Referent & \\
\hline & Yes & $17(81.82)$ & $\begin{array}{l}1.51(0.23- \\
3.14)\end{array}$ & 0.104 & $\begin{array}{l}0.61(0.29- \\
1.42)\end{array}$ & 0.252 \\
\hline \multirow{2}{*}{$\begin{array}{l}\text { Gestation } \\
\text { weeks }\end{array}$} & $\geq 37$ & 19(69.70) & 1 & & Referent & \\
\hline & $<37$ & $14(42.42)$ & $\begin{array}{l}1.69(0.76- \\
3.73)\end{array}$ & 0.194 & $\begin{array}{l}0.60(0.25- \\
1.44)\end{array}$ & 0.257 \\
\hline \multirow[t]{2}{*}{ Marital status } & Married & $29(87.88)$ & 1.0 & & Referent & \\
\hline & Unmarried & $4(12.12)$ & $\begin{array}{l}2.64(0.66- \\
10.5)\end{array}$ & 0.166 & $\begin{array}{l}1.49(0.34- \\
6.41)\end{array}$ & 0.593 \\
\hline
\end{tabular}

PPE: Proteinuric Pre-eclampsia, cOR: crude Odds Ratio, aOR: Adjusted Odds Ratio

The frequency of end-organ dysfunction of pregnant women stratified by the proteinuria status is shown in Table 3. Majority of women presented with cerebral end-organ dysfunction in both groups 108/134 $(80.1 \%)$ while haematological dysfunction was the least occurring in $18.6 \%(n=25)$. Overall, at least one organ dysfunction occurred in $28 / 33$ (84.85\%) of the women with non-proteinuric versus $92 / 101$ $(91.09 \%)$ with proteinuric pre-eclampsia: Chi-square statistic $(\chi 2)=1.035, p=0.309$.

Table 3 Comparative frequency of end-organ dysfunction between nonproteinuric and proteinuric preeclampsia at Mbarara Regional Referral Hospital 


\begin{tabular}{|c|c|c|c|c|c|c|}
\hline \multicolumn{2}{|c|}{$\begin{array}{l}\text { End-organ dysfunction } \\
\mathrm{N}=134\end{array}$} & \multirow[t]{2}{*}{ Total } & \multirow{2}{*}{$\begin{array}{l}\text { Non-proteinuric pre- } \\
\text { eclampsia } \\
\mathrm{n}(\%)\end{array}$} & \multirow{2}{*}{$\begin{array}{l}\text { Proteinuric pre- } \\
\text { eclampsia } \\
\text { n (\%) }\end{array}$} & & \multirow[t]{2}{*}{$\begin{array}{l}\mathrm{p}- \\
\text { value }\end{array}$} \\
\hline & & & & & $D f=1$ & \\
\hline \multirow[t]{2}{*}{ Multi-organ } & Yes & 120 & $28(84.85)$ & $92(91.09)$ & \multirow[t]{2}{*}{1.0353} & \multirow[t]{2}{*}{0.309} \\
\hline & No & 14 & $5(15.15)$ & $9(8.91)$ & & \\
\hline \multirow[t]{2}{*}{ Cerebral } & Yes & 108 & $25(75.76)$ & $83(82.18)$ & \multirow[t]{2}{*}{0.6557} & \multirow[t]{2}{*}{0.418} \\
\hline & No & 26 & $8(24.24)$ & 18(17.82) & & \\
\hline \multirow[t]{2}{*}{ Hepatic } & Yes & 38 & $9(27.27)$ & $29(28.71)$ & \multirow[t]{2}{*}{0.0254} & \multirow[t]{2}{*}{0.873} \\
\hline & No & 97 & $24(72.73)$ & $73(71.29)$ & & \\
\hline \multirow[t]{2}{*}{ Haematologic } & Yes & 25 & $7(21.21)$ & 18(17.82) & \multirow[t]{2}{*}{0.1884} & \multirow[t]{2}{*}{0.664} \\
\hline & No & 109 & $26(78.79)$ & 83(82.18) & & \\
\hline \multirow[t]{2}{*}{ Renal } & Yes & 34 & $8(24.24)$ & $26(25.74)$ & \multirow[t]{2}{*}{0.0296} & \multirow[t]{2}{*}{0.863} \\
\hline & No & 100 & $25(75.76)$ & $75(74.26)$ & & \\
\hline
\end{tabular}

\section{Discussion}

This study revealed a high prevalence of non-proteinuric pre-eclampsia among women admitted with preeclampsia to the maternity ward of Mbarara Regional Referral Hospital, Southwestern Uganda. Our finding compares well to the 24.9 per cent prevalence reported by Pyne et al [22] from a study conducted in Canada, and another by Homer and colleagues conducted at three tertiary hospitals in Sydney Australia [23] that reported a 26 per cent prevalence. To the contrary, our reported prevalence is lower than 38 per cent described among patients who presented with an eclamptic fit in the United Kingdom [27]. Given that most of these studies on non-proteinuric pre-eclampsia were based in high-income countries, our study provides new insights into the burden of non-proteinuric pre-eclampsia in low-income countries. Our finding thus adds pragmatic evidence to potentially improve maternal-fetal outcomes by detecting pre-eclampsia cases that would otherwise be missed by the traditional diagnostic criteria [28].

In this study, being primigravid was almost threefold associated with non-proteinuric pre-eclampsia, similar to what was reported by Omenya and colleagues at Kisii Teaching and Referral Hospital, Kisii County, Kenya [29]. Broadly, literature reports increased risk of pre-eclampsia in Europe [30] India [6] and Africa[31] among primigravidae. Pre-eclampsia prevalence of up to 3.9 per cent among first pregnancies compared to 1.7 per cent, and 1.8 per cent for second and third pregnancies respectively [32] was reported by Skjærven and colleagues in Norway. Our finding is also supported by epidemiological data showing a lower incidence of pre-eclampsia in subsequent pregnancies or with increased duration of sexual activity before pregnancy and loss of this protective effect if there is a change in paternity and among women using barrier methods of contraception [33]. The feasible explanation for this result 
relates to the role of immunology in the pathogenesis of pre-eclampsia. The immune system of primigravidae may lack adequate exposure to paternal antigens making them deficient of the protective advantage of desensitization.

We found no significant difference in the frequency of the end-organ dysfunction among women with non-proteinuric and proteinuric pre-eclampsia. We think women without proteinuria are not different from those with pre-eclampsia-in agreement with the expert review by Bartal and colleagues on proteinuria during pregnancy [34]. Moreover, the proportions of end-organ dysfunction did not differ within the proteinuric pre-eclampsia. Our results also well-align with those from a study conducted at a tertiary hospital in Sydney, Australia that showed no difference in adverse maternal outcome in relation to their proteinuria status [22]. We agree with the International Society for the Study of Hypertension in Pregnancy (ISSHP) report that defines pre-eclampsia as sustained hypertension developing after 20 weeks of gestation with maternal end-organ dysfunction-including renal, hepatic, haematological, neurological complications, or fetal growth restriction with or without proteinuria $[7,35]$.

While our study informs clinical practice on the prevalence of and factors associated with non-proteinuric pre-eclampsia as well as the frequency of end-organ dysfunction at a tertiary hospital in a resourcelimited setting, it was not without limitations. First, we measured urine protein once using urine dipstick at admission in keeping with the routine clinical practice which might have underestimated the patients with non-proteinuric preeclampsia. Second, clinically diagnosed cerebral dysfunction may have led to overreporting the cases of cerebral end-organ damage. And third, the study was conducted at a single site and therefore might not be generalized to all other regional referral hospitals.

\section{Conclusion}

Our study findings imply that non-proteinuric pre-eclampsia is common among women with preeclampsia at a tertiary hospital in southwestern Uganda especially among the primigravidae. Indifference in end-organ dysfunction frequency between women with non-proteinuric and proteinuric pre-eclampsia means that proteinuria alone may not be a pertinent piece of information in the HDP care cascade. We recommended that clinical care teams should increase surveillance of HDP, especially among first-time pregnant women. Further research should qualitatively assess providers perspectives as well as barriers to diagnosis and management of non-proteinuric pre-eclampsia.

\section{Abbreviations}

FIGO International Federation of Gynecology and Obstetrics

HDP Hypertensive Disorders of Pregnancy

ISSHP International Society for the Study of Hypertension in Pregnancy

MUST Mbarara University of Science and Technology 
MRRH Mbarara Regional Referral Hospital

PPE Proteinuric Pre-eclampsia

REC Research Ethics Committee

\section{Declarations}

\section{Ethics approval and consent to participate}

We obtained ethical approval for this study from the Institutional Review Board of Mbarara University of Science and Technology Research Ethics Committee (MUST REC); Protocol reference number: 21/01-19. Informed consent was obtained from all adults aged 18 years and over. Informed consent was also obtained from parents or legal guardian of study participants aged below 18 years. All study methods were performed in accordance with the Declaration of Helsinki guidelines and regulations.

\section{Consent for publication}

Not applicable

\section{Availability of data and materials}

De-identified data sufficient to produce primary study findings will be made available on reasonable request to the Department of Obstetrics and Gynecology, Mbarara University of Science and Technology. Data requests can be submitted through the corresponding author.

\section{Competing interests}

We declare no competing interests

\section{Authors' contributions}

AO conceived the study. AO, HML, WGMS made contributions to the study design and manuscript writing. LT, MK, GT and JN contributed towards methodology and manuscript writing. All authors substantially participated in data analysis and interpretation. All authors reviewed and approved the final manuscript.

\section{Acknowledgements}

We acknowledge the Director of Mbarara Regional Referral Hospital for allowing us to conduct this study at the hospital. We are also grateful to our research assistant, Patience Naiga, for helping with data collection and our study participants.

Preliminary results from this study were presented as an abstract during the International Federation of Gynecology and Obstetrics (FIGO) Africa Kigali congress 2020. The abstract was published in the FIGO journal special issue and is available at https://obgyn.onlinelibrary.wiley.com/doi/10.1002/ijgo.13438 


\section{Authors' information (optional)}

Not applicable.

\section{References}

1. ACOG, C.o.O.P., ACOG practice bulletin. Diagnosis and management of preeclampsia and eclampsia. Number 33, January 2002. American College of Obstetricians and Gynecologists. International journal of gynaecology and obstetrics: the official organ of the International Federation of Gynaecology and Obstetrics, 2002. 77(1): p. 67.

2. Ngonzi, J., et al., Puerperal sepsis, the leading cause of maternal deaths at a Tertiary University Teaching Hospital in Uganda. BMC pregnancy and childbirth, 2016. 16(1): p. 207.

3. MoH, U., Uganda Clinical Guidelines 2016 National Guidelines for Management of Common Conditions Health, Editor. 2016: Kampala.

4. Eiland, E., C. Nzerue, and M. Faulkner, Preeclampsia 2012. Journal of pregnancy, 2012. 2012.

5. Lindheimer, M.D. and D. Kanter, Interpreting abnormal proteinuria in pregnancy: the need for a more pathophysiological approach. Obstetrics \& Gynecology, 2010. 115(2): p. 365-375.

6. Aabidha, P.M., et al., Maternal and fetal outcome in pre-eclampsia in a secondary care hospital in South India. Journal of family medicine and primary care, 2015. 4(2): p. 257.

7. Tranquilli, A., et al., The classification, diagnosis and management of the hypertensive disorders of pregnancy: a revised statement from the ISSHP. Pregnancy hypertension, 2014. 4(2): p. 97.

8. Visintin, C., et al., Management of hypertensive disorders during pregnancy: summary of NICE guidance. Bmj, 2010. 341: p. c2207.

9. Brown, M.A., et al., Hypertensive disorders of pregnancy: ISSHP classification, diagnosis, and management recommendations for international practice. Hypertension, 2018. 72(1): p. 24-43.

10. Steegers, E.A., et al., Pre-eclampsia. The Lancet, 2010. 376(9741): p. 631-644.

11. Maric-Bilkan, C., et al., Research recommendations from the National Institutes of Health workshop on predicting, preventing, and treating preeclampsia. Hypertension, 2019. 73(4): p. 757-766.

12. Magee, L.A., et al., The FIGO Textbook of Pregnancy Hypertension: An Evidence-based Guide to Monitoring, Prevention and Management: Incorporating the Key Findings of the PRE-EMPT Global Studies. 2016: Global Library of Women's Medicine.

13. Sibai, B.M., Diagnosis, controversies, and management of the syndrome of hemolysis, elevated liver enzymes, and low platelet count. Obstetrics \& Gynecology, 2004. 103(5): p. 981-991.

14. Sibai, B.M., Eclampsia: VI. Maternal-perinatal outcome in 254 consecutive cases. American journal of obstetrics and gynecology, 1990. 163(3): p. 1049-1054.

15. Helewa, M.E., et al., Report of the Canadian Hypertension Society Consensus Conference: 1. Definitions, evaluation and classification of hypertensive disorders in pregnancy. Cmaj, 1997. 157(6): p. 715-725. 
16. Giguere, Y., et al., Combining biochemical and ultrasonographic markers in predicting preeclampsia: a systematic review. Clinical chemistry, 2010. 56(3): p. 361-375.

17. Ayele, G., S. Lemma, and E. Agedew, Factors Associated with Hypertension during Pregnancy in Derashie Woreda South Ethiopia, Case Control. Quality in Primary Care, 2016. 24(5): p. 207-213.

18. Kiondo, P., et al., Adverse neonatal outcomes in women with pre-eclampsia in Mulago Hospital, Kampala, Uganda: a cross-sectional study. The Pan African medical journal, 2014. 17(Suppl 1).

19. Melese, M.F., M.B. Badi, and G.L. Aynalem, Perinatal outcomes of severe preeclampsia/eclampsia and associated factors among mothers admitted in Amhara Region referral hospitals, North West Ethiopia, 2018. BMC research notes, 2019. 12(1): p. 1-6.

20. Duckitt, K. and D. Harrington, Risk factors for pre-eclampsia at antenatal booking: systematic review of controlled studies. Bmj, 2005. 330(7491): p. 565.

21. Bartsch, E., et al., Clinical risk factors for pre-eclampsia determined in early pregnancy: systematic review and meta-analysis of large cohort studies. Bmj, 2016. 353.

22. Payne, B., et al., PIERS proteinuria: relationship with adverse maternal and perinatal outcome. Journal of Obstetrics and Gynaecology Canada, 2011. 33(6): p. 588-597.

23. Homer, C.S., et al., Non-proteinuric pre-eclampsia: a novel risk indicator in women with gestational hypertension. Journal of hypertension, 2008. 26(2): p. 295-302.

24. von Dadelszen, P., et al., Preeclampsia in low and middle income countries-health services lessons learned from the PRE-EMPT (PRE-eclampsia-eclampsia monitoring, prevention \& treatment) project. Journal of Obstetrics and Gynaecology Canada, 2012. 34(10): p. 917-926.

25. Lugobe, H.M., et al., Risks of adverse perinatal and maternal outcomes among women with hypertensive disorders of pregnancy in southwestern Uganda. PloS one, 2020. 15(10): p. e0241207.

26. Harris, P.A., et al., Research electronic data capture (REDCap)-a metadata-driven methodology and workflow process for providing translational research informatics support. Journal of biomedical informatics, 2009. 42(2): p. 377-381.

27. Douglas, K. and C. Redman, Eclampsia in the united kingdom. Bmj, 1994. 309(6966): p. 1395-1400.

28. ACOG, Hypertension in pregnancy. Report of the American College of Obstetricians and Gynecologists' task force on hypertension in pregnancy. Obstetrics and gynecology, 2013. 122(5): p. 1122.

29. Omenya, E.R., et al., Factors contributing to Hypertension in Pregnancy among mothers attending Antenatal Clinic in Kisii Teaching and Referral Hospital, Kisii County, Kenya. World Journal of Innovative Research, 2018. 4(3): p. 262492.

30. Hutcheon, J.A., S. Lisonkova, and K. Joseph, Epidemiology of pre-eclampsia and the other hypertensive disorders of pregnancy. Best practice \& research Clinical obstetrics \& gynaecology, 2011. 25(4): p. 391-403.

31. Meazaw, M.W., et al., Factors associated with hypertensive disorders of pregnancy in sub-Saharan Africa: A systematic and meta-analysis. PloS one, 2020. 15(8): p. e0237476. 
32. Skjærven, R., A.J. Wilcox, and R.T. Lie, The interval between pregnancies and the risk of preeclampsia. New England Journal of Medicine, 2002. 346(1): p. 33-38.

33. Taylor, R.N., et al., Chesley's hypertensive disorders in pregnancy. 4th ed. 2014: Elsevier.

34. Fishel Bartal, M., M.D. Lindheimer, and B.M. Sibai, Proteinuria during pregnancy: definition, pathophysiology, methodology, and clinical significance. American Journal of Obstetrics and Gynecology, 2020.

35. Payne, B., L.A. Magee, and P. von Dadelszen, Assessment, surveillance and prognosis in preeclampsia. Best practice \& research Clinical obstetrics \& gynaecology, 2011. 25(4): p. 449-462.

\section{Figures}

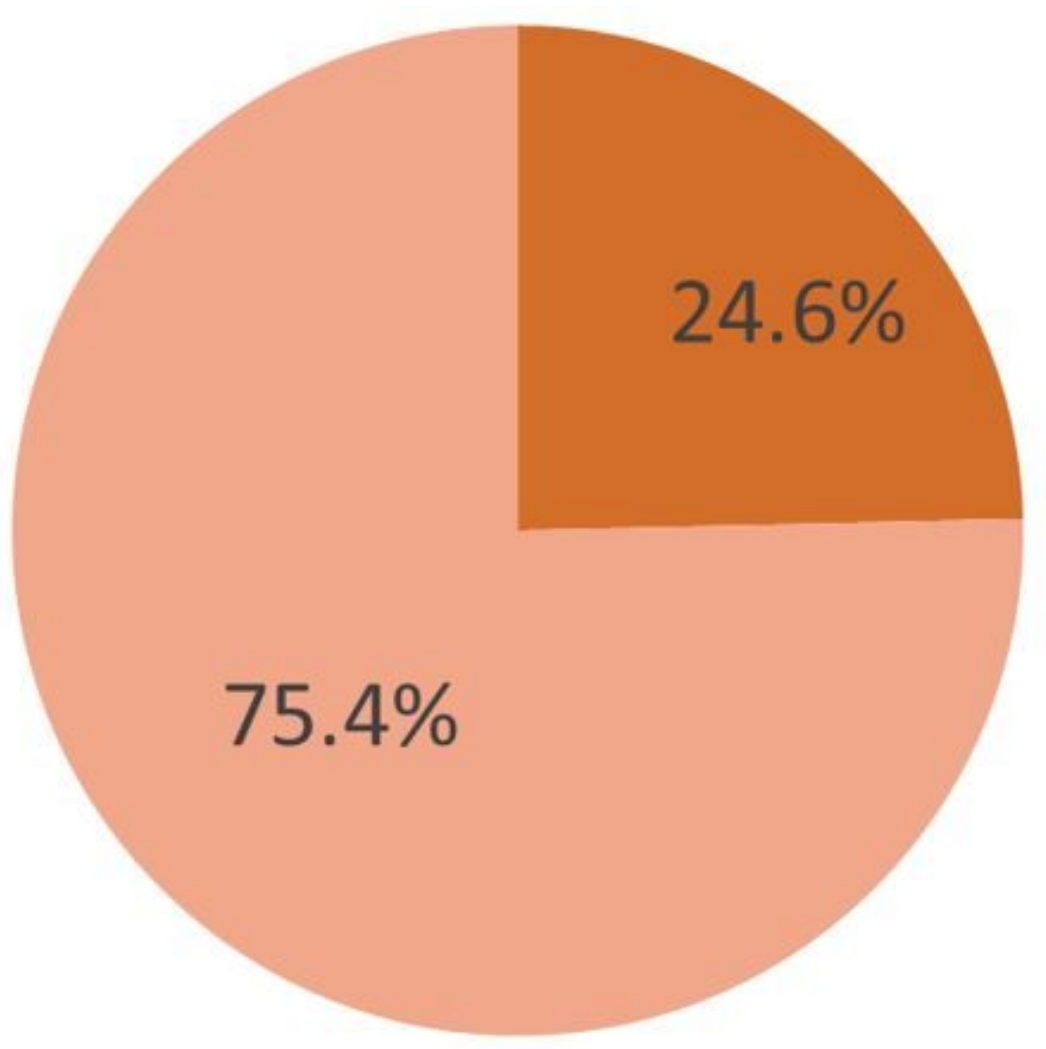

\section{Nonproteinuric Proteinuric}

\section{Figure 1}

A pie chart showing the prevalence of non-proteinuric pre-eclampsia at Mbarara Regional Referral Hospital

\section{Supplementary Files}


This is a list of supplementary files associated with this preprint. Click to download.

- NonProteinuricPreeclampsiaDataCollectiontool.pdf 